\title{
A SIMPLE FORMULATION FOR VISCO-HYPERELASTIC BEHAVIOR FOR SOFT MATERIALS SUITABLE FOR DIFFERENT LOADING TYPES
}

\author{
Olatz García Bañales ${ }^{1}$, Luis Saucedo Mora ${ }^{1,2}$, José María Benítez Baena ${ }^{1}$, Francisco \\ Javier Montáns ${ }^{1,3}$ \\ ${ }^{1}$ Universidad Politécnica de Madrid \\ ETS de ingeniería Aeronáutica y del Espacio, Pza Cardenal Cisneros 3, 28040 Madrid \\ ${ }^{2}$ Department of Materials, University of Oxford \\ Parks Road, Oxford, OX1 3PJ, UK \\ ${ }^{3}$ Department of Mechanical and Aerospace Engineering, Herbert Wertheim College of Engineering \\ University of Florida, FL 32611, USA
}

Key words: Viscoelasticity, hyperelasticity, soft materials

\begin{abstract}
Soft materials, and especially soft biological tissues, have a complex highly nonlinear behavior both for quasielastic (slow) and viscous loading. In partiular, the cyclic behavior is different depending on the loading speed, number of cycles, and their magnitude. Furthermore, different soft materials and soft tissues have different particularities in their behavior. Therefore, a phenomenological proposal capable of accurately capturing all these singularities with few, easy to obtain parameters based on experimental data, is valuable. In this study, a visco-hyperelastic one-dimensional formulation to characterize different biological tissues is proposed, which has proven to be capable of capturing the response of numerous soft biological tissues (brain tissue, coronary arteries, tendons, tongue tissues, abdominal muscle, cells...) under pure and combined loading modes, including tension, compression, simple shear and the combination of the latter one with tension and compression. One of the main advantages of the proposed model is its simplicity, being that the formulation is calibrated with four simple parameters (two of them for the hyperelastic component and four for dealing with the different viscous aspects) obtained from the uniaxial loading. The formulation, based on a combination of Maxwell and Kelvin-Voigt rheological models has proven to represent to very good accuracy the behavior of a wide range of materials under different types of loadings, including effects like preconditioning and cycle stabilization. In all these cases, under different monotonic and cyclic loading, all aspects of the viscous and elastic behavior are accurately captured. Thanks to its structure, this model incorporates strain-level dependent nonequilibrium viscoelasticity and it may be easily incorporated to 3D nonlinear finite strains formulations.
\end{abstract}

\section{INTRODUCTION}

Biological tissues are extremely complex and their behavior is controlled by numerous mechanisms that make it hard to completely capture their response. As a consequence, the models developed reflect that 
complexity in the fact that it usually takes a big set of parameters, complicated formulations and finite element models to reproduce their response.

While it is extremely important to develop complex models that are able to capture all the mechanisms and phenomena in order to simulate tissue behavior as precisely as possible, it is also very useful to create simpler models that, with a reduced number of equations and almost no computational cost, can easily and rapidly make an estimation of the general characteristics of the response. Trading a small amount of accuracy for a very important amount of simplicity is what will be done in this study. Under the belief that there are no general right or wrong answers, and that every particular case needs to be studied with the appropriate means to it, this model is designed to be as simple as possible providing a very acceptable accuracy. Depending on the degree of precision needed in the computational simulation, the choice will be one or another, but it is important to have a wide range of options to cover every situation. For instance, if there is a need for an advanced study where a high precision is needed for a delicate matter, then the complex model will be the right choice. If, however, the study focuses on a basic understanding for diagnosis and the main requirement is that the time and effort are as reduced as possible but still getting a great fit, then a simpler model would be the optimal solution.

Overall, the aim of this study is to propose a simple, computationally cheap model with few parameters that accurately characterizes the general one-dimensional response of numerous biological tissues under several loading modes and conditions. It is also possible to apply it to other soft, rubber-like materials.

\section{PROPOSED MODEL}
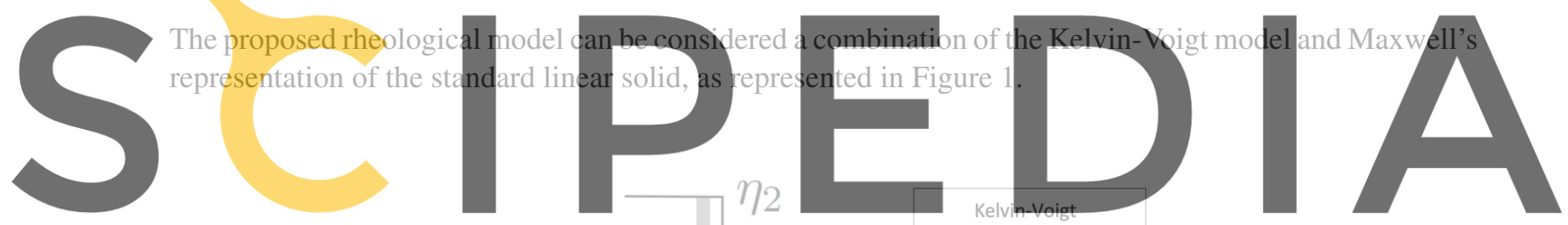

Register for free at https//www.scipedia.com to download the version without the watermark

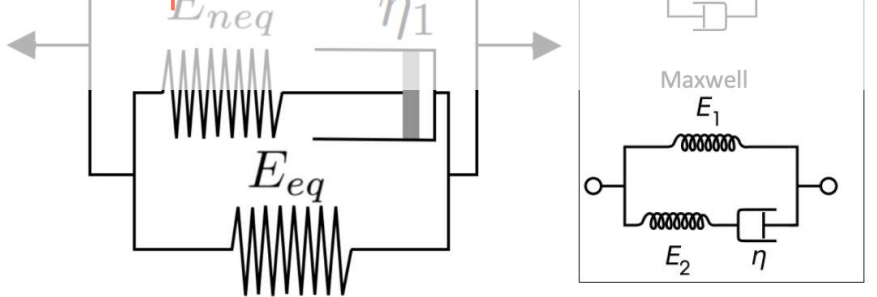

Figure 1: Proposed rheological model

The upper dashpot, with viscosity $\eta_{2}$, plays an important role in the dynamic process, being responsible for the viscous dissipation during the loading and unloading cycles, while the lower dashpot, with viscosity $\eta_{1}$, is responsible for the viscous dissipation of the non-equilibrated deformation energy.

In a dynamic test, the load is applied with a high velocity relative to the relaxation time. In addition, the characteristic time in a relaxation test is higher than the one in dynamic loading and in consequence, the 
rate of viscous deformation in the lower dashpot $\left(\eta_{1}\right)$ can be considered constant during cyclic loading. This fact can also be seen as if the viscous effect of the non-equilibrated dashpot, $\eta_{1}$, is frozen.

When subjected to uniaxial loading, the stress according to this model can be expressed as

$$
\sigma=\sigma^{e q}+\sigma^{n e q}+\sigma^{v}
$$

Where $\sigma^{e q}$ is the equilibrated stress, $\sigma^{n e q}$ is the non-equilibrated stress and $\sigma^{v}$ is the stress in the upper dashpot, $\eta_{2} . \sigma^{e q}$ and $\sigma^{\text {neq }}$ are obtained by taking derivatives of the strain energy function with respect to the corresponding stretch.

\subsection{Infinitesimal strains}

For infinitesimal strains, the elastic constant, $E$, in the SLS model is identified as the Young's modulus. Considering that both springs have the same Young's modulus $E=E_{e q}=E_{n e q}$, the equilibrated and non-equilibrated stresses can be obtained as

$$
\sigma^{e q}=E \varepsilon
$$
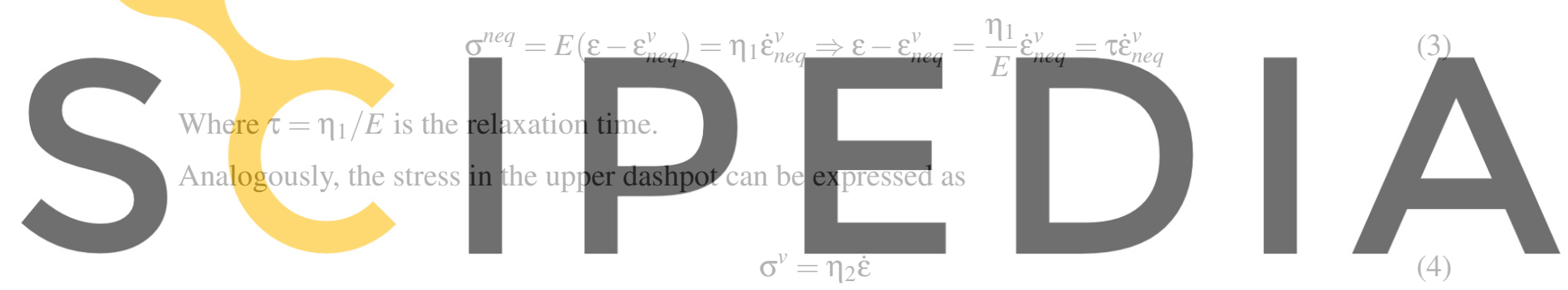

Register f\$6, freesiattindtps//Www.scipedia.com to download the version without the watermark

$$
\sigma=\sigma^{e q}+\sigma^{n e q}+\sigma^{v}=E \varepsilon+E \tau \dot{\varepsilon}_{n e q}^{v}+\eta_{2} \dot{\varepsilon}=E \varepsilon\left(1+\frac{\tau}{\varepsilon} \dot{\varepsilon}_{n e q}^{v}\right)+\eta_{2} \dot{\varepsilon}
$$

And finally

$$
\sigma=E \varepsilon\left(1+\xi \dot{\varepsilon}_{n e q}^{v}\right)+\eta_{2} \dot{\varepsilon}
$$

Where $\xi=\tau / \varepsilon$ is assumed constant, so that the relaxation time depends on the level of deformation.

\subsection{Relaxation tests}

In relaxation tests, the strain is mantained so that $\dot{\varepsilon}=0$ and Eq. 6 is written as

$$
\sigma=E \varepsilon\left(1+\xi \dot{\varepsilon}_{n e q}^{v}\right)
$$


In this case, the strain energy stored in the equilibrated part is constant, the part that remains after the stress-time relaxation curve reaches the asymptotic behavior. In contrast, the one stored in the nonequilibrated spring is dissipated due to the activation of the dashpot and the consequent viscous strain. Initially, the dashpot is activated with a certain strain rate that decreases with time so that, according to Eq. 7, the stress also does. This process is finished when $\dot{\varepsilon}_{\text {neq }}^{v} \approx 0$ and the stationary regime is reached. In this state the material has relaxed and $\sigma=\sigma^{e q}$.

\subsection{Dynamic loading}

As mentioned, in loading and unloading cycles, the term $\hat{\xi}=\xi \dot{\varepsilon}_{n e q}^{v}$ is considered to remain constant and therefore, it will be determined as a material parameter.

In this study, a certain viscoelastic material is considered to be subjected to a sinusoidal state of deformation in the form of

$$
\lambda=\left(\lambda_{\max }-1\right) \sin (\omega t)+1
$$

\section{Replacing the value of the sinusoidal stretch and its derivative in Eq. 2}

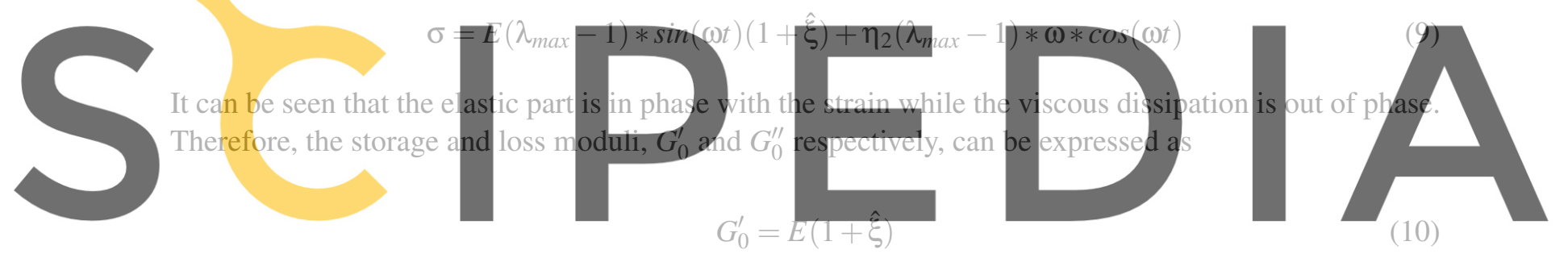

Register for free at https//www.scipedia.com to download the version without the watermark

$$
G_{0}^{\prime \prime}=\eta_{2} \omega \cot (\omega t)
$$

\subsection{Application to finite strains}

Starting from the formulation for infinitesimal strains and taking into account that the shape of the stressstrain curves of hyperelastic materials can be compared to the curve of a tangent (see Figure 2), in this model we proposed to turn the linear strain into nonlinear strain through the tangent function.

The elastic part of the stress can be written as

$$
\sigma=E \frac{2}{\pi}\left(\lambda_{\text {lim }}-1\right) \tan \left(\frac{\pi}{2} \frac{\lambda-1}{\lambda_{\text {lim }}-1}\right)
$$

Where $\lambda_{\text {lim }}$ is the asymptote of the tangent that physically represents the maximum deformation that the material can develop. 


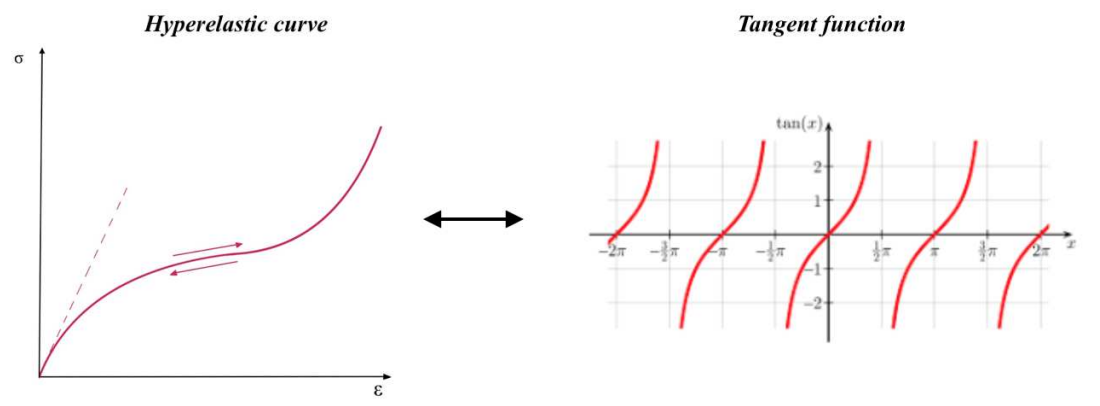

Figure 2: Similarity between the hyperelastic stress-strain curve and tangent function

This asymptotic behavior is compatible with the microscopic behavior of hyperelastic materials like rubbers or biological tissue, since it represents the lock of chains, synthetic or biological, that are the microscopic constituents of this kind of materials.

In finite strains, $E$ is no longer the Young's modulus because the material's behavior is not linear anymore. Therefore, the elastic modulus will be expressed as $\kappa$ and it is a hyperelastic parameter that will be determined from experimental results.

The model for finite strains can be considered an extension of the one for infinitesimal strains, since

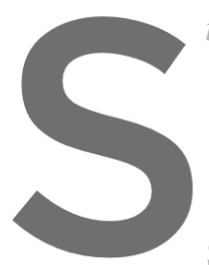
$\tan (x) \approx x$ when $x \ll 1$, then

So, the stress for infinitesimal strains can be writter
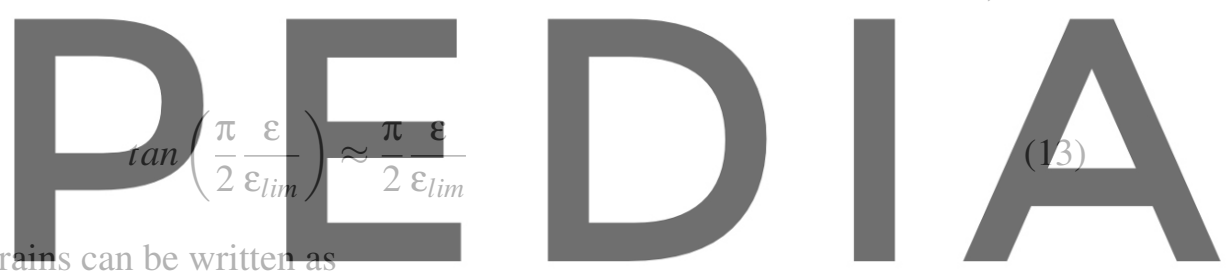

Register for free at https//www.scipedia.comR to dowvload the version without the watermark

For finite strains and accounting for the viscous behavior

$$
\sigma=\kappa(1+\hat{\xi}) \frac{2}{\pi}\left(\lambda_{\text {lim }}-1\right) \tan \left(\frac{\pi}{2} \frac{\lambda-1}{\lambda_{\text {lim }}-1}\right)+\eta_{2} \dot{\lambda}
$$

Particularizing the equation for the sinusoidal loading case

$$
\begin{array}{r}
\sigma=\kappa(1+\hat{\xi}) \frac{2}{\pi}\left(\lambda_{\text {lim }}-1\right) \tan \left(\frac{\pi}{2} \frac{\left(\lambda_{\text {max }}-1\right) \sin (\omega t)}{\lambda_{\text {lim }}-1}\right)+ \\
\quad+\eta_{2}\left(\lambda_{\text {max }}-1\right) \cdot \omega \cdot \cos (\omega t) \cdot \sec \left(\frac{\pi}{2} \frac{\left(\lambda_{\text {max }}-1\right) \sin (\omega t)}{\lambda_{\text {lim }}-1}\right)^{2}
\end{array}
$$


Where

$$
\hat{\xi}=\xi \dot{\varepsilon}_{n e q}^{v}=\eta_{1} \frac{2}{\pi} \omega\left(\lambda_{\text {max }}-1\right)
$$

\section{OBJECTS OF STUDY}

Due to the scarce availability of experimental tests carried out in human brain tissue, and to the difficulties to conduct an experiment, experimental data was obtained from tests available in the literature [3] [2] [1] [12] [10] [4] [8] [6] [11] [5] [9]. Several biological tissues were studied, so as to prove that the model is capable of adapting to numerous soft tissues, not only to brain samples.

The studied tissues are:

- Human brain from two different regions, from which one belongs to gray matter (cortex), and one belongs to white matter (corona radiata) [3] [2] [1].

- Porcine coronary arteries from two different locations (distal and proximal regions) in two directions each (longitudinal and circumferential)[8] [6].

- Human patellar tendon for younger and older age groups [10].

- Bovine tongue tissues, consisting of the top mucous tissue covering the tongue muscle, and the superior longitudinal muscle in three different directions of fiber orientation $\left(0^{\circ}, 20^{\circ}, 35^{\circ}\right)$ [12].
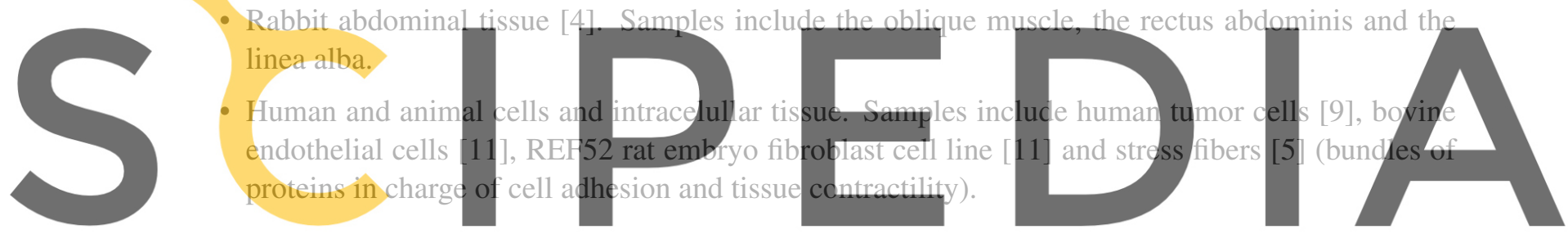

4 RESULTS

Register for free at https//www scipedia, com to down load the version without the watermark

by the proposed model and the coefficient of determination, $R^{2}$, as a representation of the goodness of fit.

\subsection{Brain tissue}

The experimental results analyzed in this section were obtained from [1], [2] and [3]. Brain tissue has the broader variety of experimental results out of all the tissues that are studied.

\section{- Conditioned viscoelastic response}

The conditioned response is evaluated, this is, the sample has already undergone two previous loading cycles containing a higher degree of preconditioning. The steady response is evaluated. Two different samples were studied to illustrate the possible differences between gray and white matter. From gray matter the cortex was analyzed and from white matter the corona radiata. 

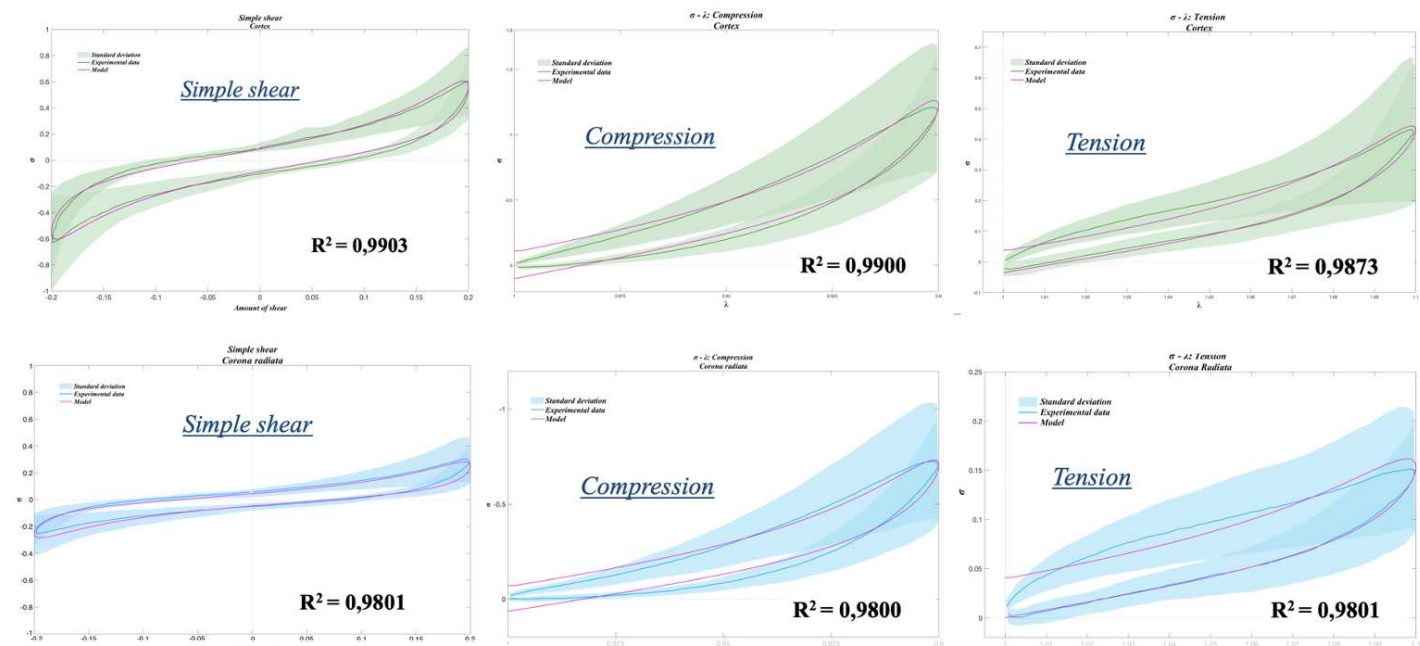

Figure 3: Conditioned viscoelastic response. Gray matter, cortex (green); white matter, corona radiata (blue)

\section{- Conditioned elastic response}

In this section the elastic response is evaluated, without the viscous part, leaving only two parameters, $\kappa$ and $\lambda_{\max } / \lambda_{\text {lim }}$ (or aos $\max /$ aos lim $_{\text {lim }}$ for shear tests). The samples are also from the cortex and

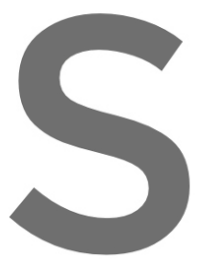
the corona radiata rather than separatin
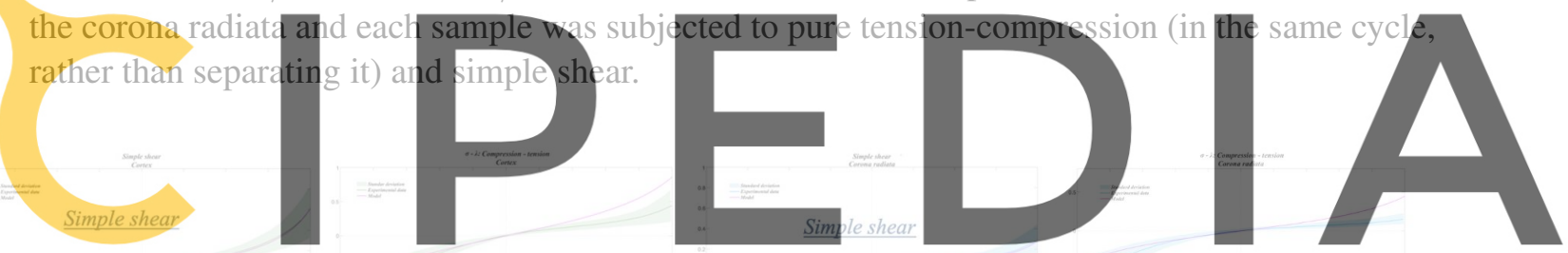

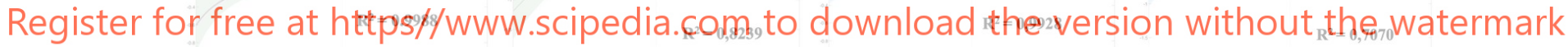

Figure 4: Conditioned elastic response. Gray matter (green), white matter (blue)

Brain tissue presents an asymmetry in tension and compression, showing a stiffer response in the latter. The proposed model, due to the limited number of parameters, is not able to capture that asymmetry with the same set of parameters. When testing tension and compression separately, with two different sets of parameters, as done in the viscoelastic case, the formulation is able to capture the response accurately.

\section{- Cyclic response}

In this section, the samples are subjected to three subsequent loading cycles, without previous conditioning. As mentioned, after the first loading cycle, where the response shows preconditioning, it reaches a steady state, where the cycles tend to overlap. The samples that were subjected to the cycle loading belong to the corona radiata (white matter). 

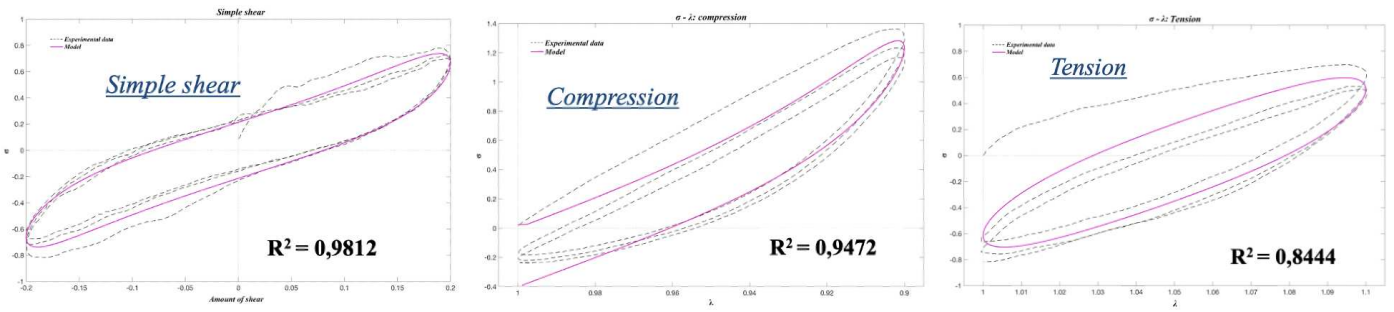

Figure 5: Cyclic viscoelastic response. White matter (corona radiata)

The model accurately characterizes all of the steady responses. In the case of tension, the preconditioning is so marked that the $R^{2}$ is penalized, but the general trend is well captured.

\section{- Viscoelastic and elastic responses to combined loading}

In this section samples of the corona radiata are studied [1]. Instead of subjecting the samples to pure loads, the loading modes are combined: sinusoidal simple shear is applied along with a certain amount of axial stretch. The studied cases are simple shear with $10 \%$ and $20 \%$ compression and tension. The elastic and viscoelastic steady response is studied, with no preconditioning.
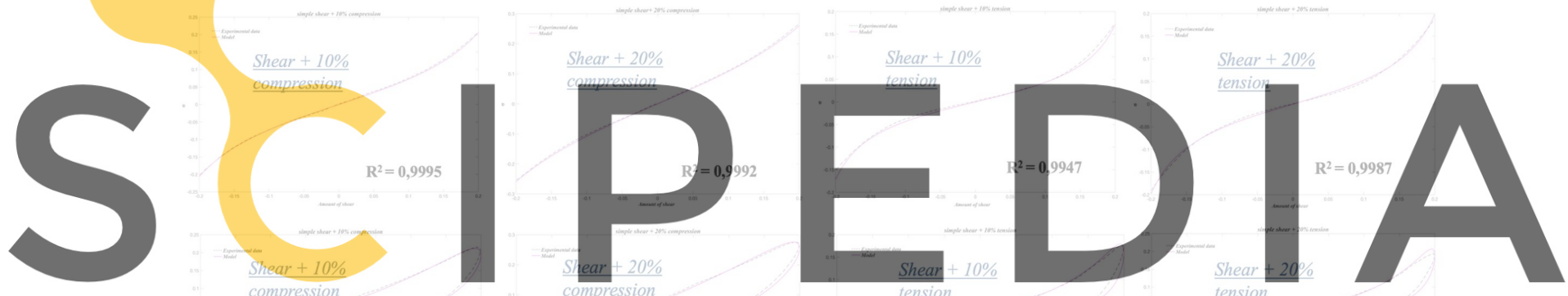

Register for free at https//www.scipedia.com to $\underset{\mathrm{R}^{2}=0,9963}{\mathrm{R}^{2}=0,9975}$

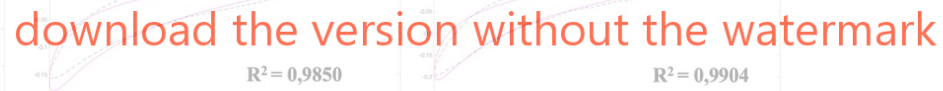

Figure 6: Elastic (upper) and viscoelastic (lower) response to combined loading. White matter (corona radiata)

All of the situations are well characterized due to the versatility of the parameters.

\subsection{Coronary arteries}

With the hope of proving that the proposed model is not only suitable for brain tissue, other biological materials were studied. In this section, samples from porcine coronary arteries were studied, with experimental tests obtained from [8] [6]. The two regions indicated in Figure ?? were tested: proximal and distal regions and, to study if directional variations affect the result, longitudinal and circumferential directions.

The proposed model successfully captures the material's response, even though the stiffness is higher by two orders of magnitude. It yields very high values of $R^{2}$ for every region and direction. 


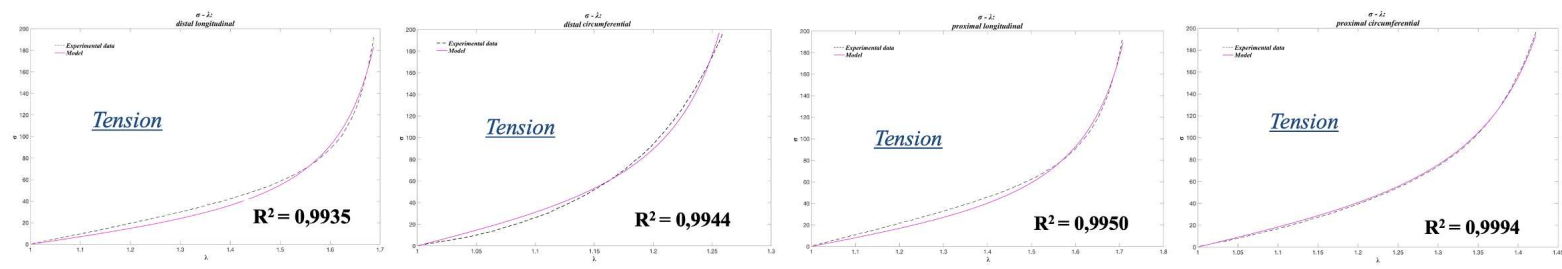

Figure 7: Elastic response to axial stretch in coronary arteries: distal region, longitudinal direction (left); distal region, circumferential direction (second left); proximal region, longitudinal direction (second right); proximal region, circumferential direction (right)

\subsection{Patellar tendon}

The present object of study is the human patellar tendon, with tests obtained from [6]. Samples are divided in two groups according to age and are subjected to tension.

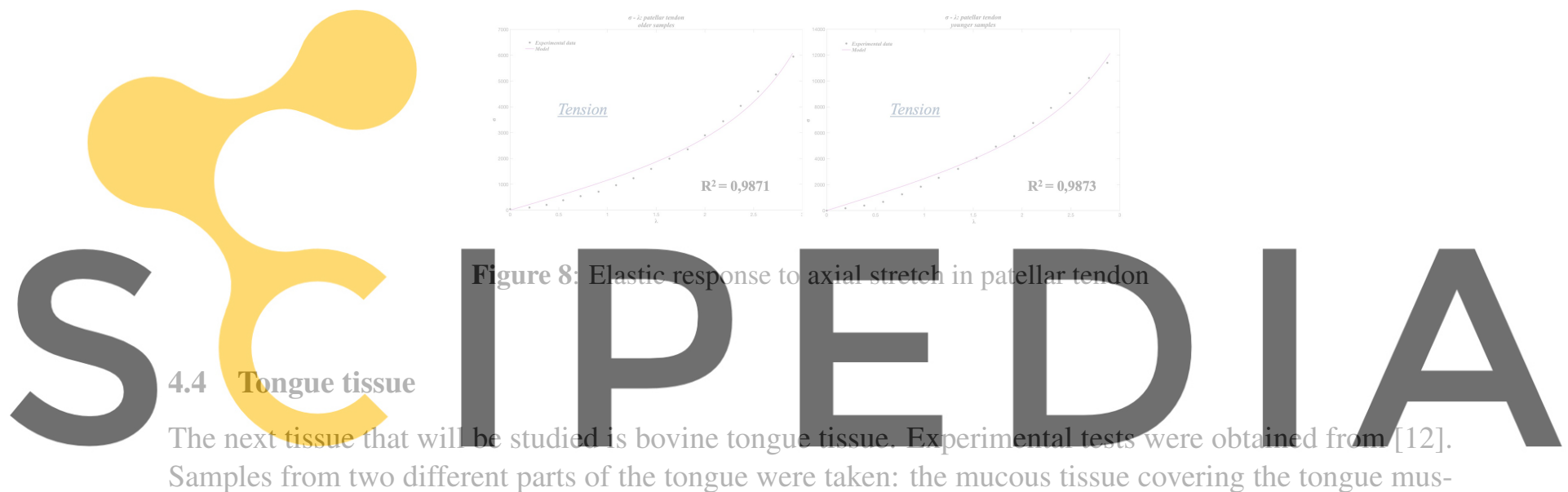

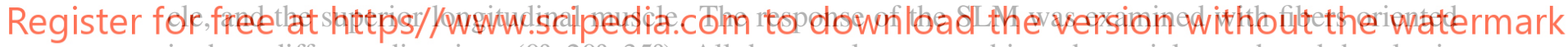

in three different directions $\left(0^{\circ}, 20^{\circ}, 35^{\circ}\right)$. All the samples were subjected to axial stretch and the elastic

response is presented.

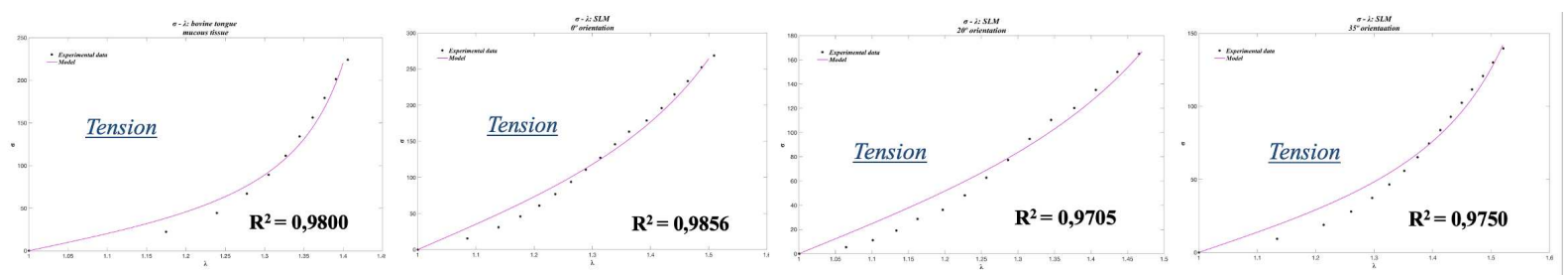

Figure 9: Elastic response to axial stretch in tongue tissue: mucous tissue (left), superior longitudinal muscle

As it could be expected, the sample is stiffer in the direction of the fibers, decreasing as the orientation is closer to the transversal direction, being congruent with the hypothesis of transverse isotropy. 


\subsection{Abdominal tissue}

Abdominal tissue presents a highly nonlinear, transversely isotropic behavior. Experimental tests were taken from [4]. All the samples were subjected to axial stretch. Congruent with the transverse isotropy, the samples show a stiffer response in the longitudinal direction.

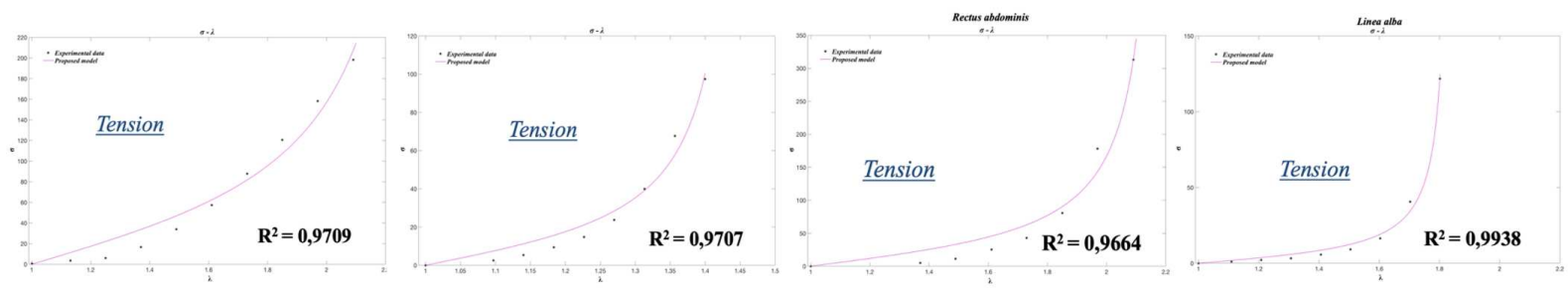

Figure 10: Elastic response to axial stretch in abdominal tissue: oblique muscle (left and second left), rectus abdominis (second right) and linea alba (right)

\subsection{Cellular level}

In order expand the model's use to the cellular level, experimental tests carried out in human and animal cells under different conditions were taken [5], [7], [9], [11].

\section{Endothelial cells}
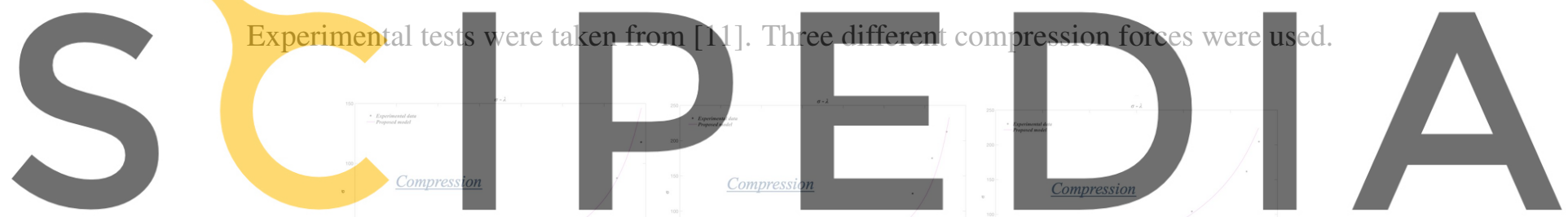

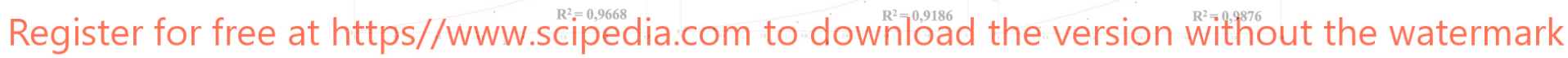

Figure 11: Elastic response to three different compression forces in bovine endothelial cells

\section{- REF52 cells}

Experimental tests for REF52 cells were obtained from [11]. REF52 is a cell line obtained from rat embryo fibroblast. The results for three different compression forces were taken.
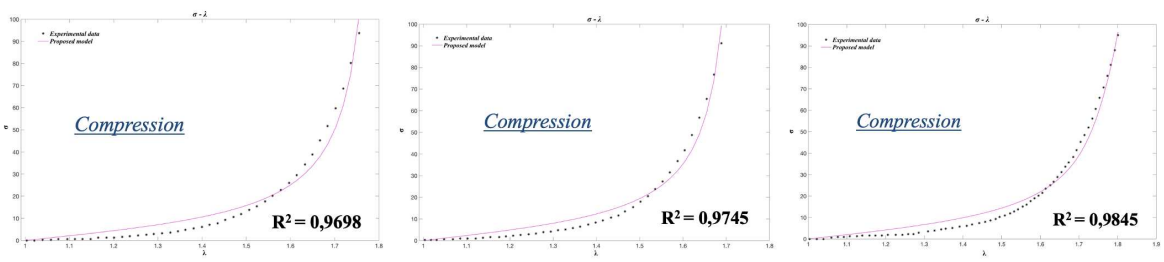

Figure 12: Elastic response to three different compression forces in REF52 cells 


\section{- Tumor cells, stress fibers and balls and springs model}

The first sample of this section comes from a malignant tumor cell. Samples were obtained from human breast cancer and the experimental tests were obtained from [9]. Cell deformation was studied and for that samples were subjected to compressive forces.

Stress fibers are contractile actin (family of proteins) bundles. Stress fibers have been proven to play an important role in cellular contractility, cell adhesion and migration. Experimental tests of isolated stress fibers are taken from [5].

Studies represent cells and their interactions with with balls and sprigs [7]. Rigid spheres represent cells and springs inside and outside these spheres account for their rigidity and tissue elasticity.
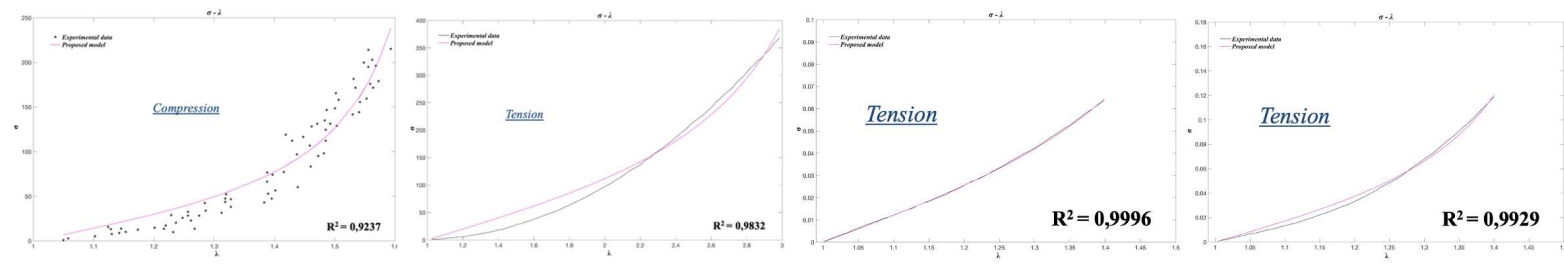

Figure 13: Elastic response to compression forces in: malignant tumor cells (left), stress fibers (second left), balls and springs model (right and second right)

\section{CONCLUSIONS}

This is a formulation that can capture the steady response of the material and it can be useful to capture the steady loads that take place during the usual functioning. Looking at Section 4 it can be stated that the objective of this study has been fulfilled. The presented formulation accurately characterizes the behavior of brain tissue under multiple loading conditions and of coronary arteries, abdominal tissue, cells, tendon, and tongue tissue under axial stretch.

There are certain aspects of the behavior that are not captured by the model, especially the preconditioning of brain tissue. This is due to the fact that the number of parameters is very limited in an attempt to make the model as simple as possible, and does not account for the biphasic or porous nature of the material. As for the tension - compression asymmetry caused by the fact that the liquid trapped inside the pores offers a higher resistence to compression than to tension, with a separate set of parameters for both cases, results are almost perfect. The proposed model yields logical, expected results in many aspects: the elastic modulus is higher for compression than for tension; also, in transversely isotropic tissues (abdominal muscle and superior longitudinal tongue muscle), the stiffness is higher in the direction of the fibers ( $0^{\circ}$ for the SLM and longitudinal direction in the abdominal case) than in transverse directions.

Leaving this mechanisms aside, the $R^{2}$ is in every case between 0.97 and 0.99 despite the changes in stiffness, deformation rate, loading mode, loading direction and region of the samples. In addition to being able to represent numerous macroscopic tissues and rubbery materials, the model can also adapt to the microscopic scale, being useful to capture the response of isolated cells subjected to compression and to model cell adhesion and tissue contractility. The parameters are very versatile and the proposed model can easily adapt to many different situations, being able to even substitute other models for cellular behavior (balls and springs models). 


\section{REFERENCES}

[1] S. Budday, G. Sommer, C. Birkl, C. Langkammer, J. Haybaeck, J. Kohnert, M. Bauer, F. Paulsen, P. Steinmann, E. Kuhl, and G.A. Holzapfel. Mechanical characterization of human brain tissue. Acta Biomaterialia, 48:319-340, January 2017.

[2] S. Budday, G. Sommer, J. Haybaeck, P. Steinmann, G.A. Holzapfel, and E. Kuhl. Rheological characterization of human brain tissue. Acta Biomaterialia, 60:315-329, September 2017.

[3] Silvia Budday, Timothy C. Ovaert, Gerhard A. Holzapfel, Paul Steinmann, and Ellen Kuhl. Fifty shades of brain: A review on the mechanical testing and modeling of brain tissue. Archives of Computational Methods in Engineering, July 2019.

[4] B. Calvo, M. Sierra, J. Grasa, M.J. Muñoz, and E. Peña. Determination of passive viscoelastic response of the abdominal muscle and related constitutive modeling: Stress-relaxation behavior. Journal of the Mechanical Behavior of Biomedical Materials, 36:47-58, August 2014.

[5] Shinji Deguchi, Toshiro Ohashi, and Masaaki Sato. Tensile properties of single stress fibers isolated from cultured vascular smooth muscle cells. Journal of Biomechanics, 39(14):2603-2610, January 2006.

[6] David González, Alberto García-González, Francisco Chinesta, and Elías Cueto. A data-driven learning method for constitutive modeling: Application to vascular hyperelastic soft tissues. Materials, 13(10):2319, May 2020.

[7] Miroslav Holeček and Fanny Moravec. Hyperelastic model of a material which microstructure is formed by "balls and springs". International Journal of Solids and Structures, 43(24):7393-7406, November 2006.

[8] Gerhard A. Holzapfel, Gerhard Sommer, Christian T. Gasser, and Peter Regitnig. Determination of layer-specific mechanical properties of human coronary arteries with nonatherosclerotic intimal thickening and related constitutive modeling. American Journal of Physiology-Heart and Circulatory Physiology, 289(5):H2048-H2058, November 2005.

[9] Shuhuan Hu, Ran Wang, Chi Man Tsang, Sai Wah Tsao, Dong Sun, and Raymond H. W. Lam. Revealing elasticity of largely deformed cells flowing along confining microchannels. RSC Advances, 8(2):1030-1038, 2018.

[10] Greg A. Johnson, Dawn M. Tramaglini, Rebecca E. Levine, Kazunori Ohno, Nam-Yong Choi, and Savio L-Y. Woo. Tensile and viscoelastic properties of human patellar tendon. Journal of Orthopaedic Research, 12(6):796-803, November 1994.

[11] Sebastian J Müller, Franziska Weigl, Carina Bezold, Christian Bächer, Krystyna Albrecht, and Stephan Gekle. A hyperelastic model for simulating cells in flow. arXiv preprint arXiv:2003.03130, 2020 .

[12] Ali-Akbar Karkhaneh Yousefi, Mohammad Ali Nazari, Pascal Perrier, Masoud Shariat Panahi, and Yohan Payan. A visco-hyperelastic constitutive model and its application in bovine tongue tissue. Journal of Biomechanics, 71:190-198, April 2018. 